\title{
Urocortin 2 induces potent long-lasting inhibition of cardiac sympathetic drive despite baroreflex activation in conscious sheep
}

\author{
Christopher J Charles, David L Jardine, Miriam T Rademaker and A Mark Richards \\ Christchurch Cardioendocrine Research Group, University of Otago, Christchurch, PO Box 4345, Christchurch 8140, New Zealand \\ (Correspondence should be addressed to C J Charles; Email: chris.charles@otago.ac.nz)
}

\begin{abstract}
Emerging evidence suggests that the urocortin (UCN) peptides contribute to pressure and volume regulation with possible involvement in the pathophysiology of cardiovascular disease. We have recently reported that i.v. UCN1 potently inhibits cardiac sympathetic nerve activity (CSNA) in normal sheep. However, little is known about possible interactions between UCN2 and the sympathetic nervous system. Accordingly, we have examined the effects of i.v. UCN2 on CSNA, hemodynamics, and plasma catecholamines in normal conscious sheep. Bolus i.v. administration of UCN2 (25 and $100 \mu \mathrm{g})$ resulted in the expected hemodynamic actions including transient falls in arterial pressure $(P=0 \cdot 016)$ and more sustained rises in heart rate $(P<0 \cdot 001)$ and cardiac output $(P<0 \cdot 001)$ and falls in peripheral resistance $(P<0 \cdot 001)$. CSNA burst frequency showed a biphasic response $(P<0 \cdot 001)$ with an acute rise
\end{abstract}

followed by a more prolonged fall. CSNA burst area and incidence showed prolonged, dose-dependent falls in response to UCN2 administration (all $P<0 \cdot 001$ ). UCN2 also induced a short-lived rise in plasma norepinephrine levels $(P=0 \cdot 006)$. The marked rise in heart rate in response to $\mathrm{UCN} 2$ is preserved in sheep undergoing pharmacological ganglionic blockade with hexamethonium. In conclusion, this is the first study to report the effects of UCN2 on SNA and indicates potent inhibition of sympathetic traffic to the heart despite a generalized baroreceptor-induced activation of sympathetic activity. These findings suggest an important role for UCN2 in cardiovascular homeostasis and warrant further investigation for potential therapeutic applications in acute myocardial injury and heart disease.

Journal of Endocrinology (2010) 204, 181-189

\section{Introduction}

Emerging evidence suggests that the urocortin (UCN) peptides UCN1, UCN2, and UCN3, recently identified members of the corticotropin-releasing factor (CRF) peptide family, contribute to pressure and volume regulation with possible involvement in the pathophysiology of cardiovascular disease (as reviewed by Charles et al. (2004), Fekete \& Zorilla (2007) and Boonprasert et al. (2008)). Unlike UCN1, which shows little selectivity for CRF receptor subtypes, UCN2 (and UCN3) binds selectively to the G protein-coupled CRF receptor type $2\left(\mathrm{CRF}_{2}\right.$; Hsu \& Hsueh 2001, Lewis et al. 2001, Reyes et al. 2001). Both UCN2 and $\mathrm{CRF}_{2}$ are widely distributed throughout the brain and peripheral tissues, with strong expression apparent in the heart and vasculature (Wiley \& Davenport 2004). We have recently reported the bioactivity of UCN1 (Rademaker et al. 2002), UCN2 (Rademaker et al. 2005) and UCN3 (Rademaker et al. 2006) in sheep with and without pacing-induced heart failure (HF). All three peptides exhibit similar bioactivity, inducing reductions in peripheral resistance, arterial and left atrial pressures and increases in cardiac output in association with attenuation of vasoconstrictor/volume-retaining neurohumoral systems. However, the time-course of effects of the three peptides differs markedly with UCN1 having a long half-life $(19 \mathrm{~h})$ with bioactivity lasting more than $24 \mathrm{~h}$ following i.v. bolus doses compared to a much shorter time-course of action (several hours) for UCN2 and UCN3 (Rademaker et al. 2002, 2005, 2006).

The sympathetic nervous system (SNS) is a pivotal element of normal cardiac and circulatory regulation. We have recently reported that UCN1 potently inhibits cardiac sympathetic nerve activity (CSNA) in normal sheep (Charles et al. 2008). However, little is known about possible interactions between the more recently identified UCN2 and the SNS. UCN2-induced rises in heart rate do not appear to be solely baroreflex mediated as, in normal sheep, UCN2 increases heart rate and blood pressure in parallel (Rademaker et al. 2005). This raises the possibility that UCN2 may modulate sympathetic outflow from the central nervous system (CNS). Studies examining the effects of UCN2 on plasma norepinephrine report either no effect (Rademaker et al. 2005, Davis et al. 2007a) or activation (Davis et al. 2007b) of norepinephrine, taken as an index of global activity of the SNS. To our knowledge, there have been no studies investigating the effect of UCN2 on SNA to a specific organ 
as measured by direct means, such as microneurography. Accordingly, we have examined the effects of UCN2 on CSNA, hemodynamics, and plasma catecholamines in normal conscious sheep.

\section{Materials and Methods}

The Animal Ethics Committee of the University of Otago, Christchurch approved the study protocol. A total of seven Coopworth ewes weighing 53-76 kg (Lincoln University Farm, Christchurch, New Zealand) were housed in an air-conditioned, light-controlled room and received a diet of lucerne chaff and food pellets providing $75 \mathrm{mmol}$ sodium and $150 \mathrm{mmol}$ potassium per day. Under general anesthesia (induced by $17 \mathrm{mg} / \mathrm{kg}$ thiopentone sodium and maintained by a mixture of halothane, nitrous oxide, and oxygen), a left lateral thoracotomy was performed to allow up to five stainless steel needle electrodes to be inserted and glued in the thoracic cardiac nerves as previously described (Jardine et al. 2002). Connecting leads were sutured to the mediastinum and exteriorized dorsally through the chest wall. During the same anesthetic, via a $5-\mathrm{cm}$ neck incision, a carotid artery was cannulated (16G Angiocath; Becton Dickinson, Sandy, UT, USA) for direct measurement of arterial pressure and heart rate; polyethylene catheters were placed in the jugular vein for blood sampling, and a Swan-Ganz thermodilution catheter (Edwards Life Sciences, Irvine, CA, USA) was placed in the pulmonary artery via the jugular vein for measurements of cardiac output. The animals were allowed to recover for at least 4 days before experiments.

CSNA recordings were made in fully conscious sheep from pairs of electrodes via a preamplifier with an active probe (DAM-80 pre-amplifier, World Precision Instruments Inc., Saratosa, FL, USA). The raw signal was amplified $\left(\times 10^{6}\right)$, filtered between 300 and $3000 \mathrm{~Hz}$, and integrated using a time constant of $100 \mathrm{~ms}$. The integrated nerve signal was digitally converted using in-house software (sampling rate $200 \mathrm{~Hz}$ ), and postganglionic efferent sympathetic activity was identified in all animals by the following characteristics: a) bursts were synchronised to the diastolic phase of the arterial pulse; b) bursts decreased during sympathetic blockade with hexamethonium infusion $(2 \mathrm{mg} / \mathrm{kg}$ over $2 \mathrm{~h}$ ) on day 3 postthoracotomy; and c) an inverse relationship existed between burst area and diastolic blood pressure during baroreflex tests undertaken on each recording day. Background noise was quantified as the small deviations from baseline observed during hexamethonium infusion and phenylephrine bolus. Only recordings with a signal-to-noise ratio of greater than two and coinciding with the correct part of the cardiac cycle were analyzed. Using these criteria on the first day, the best signal from all possible electrode combinations was selected and used for subsequent recordings. CSNA was quantified from the integrated nerve signal by: a) counting the number of bursts per minute (burst frequency); b) counting the number of bursts per 100 heart beats (burst incidence); and c) measuring the area under the integrated signal per minute (burst area/min) and burst area/100 beats.

Each animal was studied on two occasions 1-3 days apart, receiving vehicle control (10 $\mathrm{ml}$ of $0.9 \%$ saline) and murine UCN2 (American Peptides, Sunnyvale, CA, USA) according to a balanced random order design. Ovine UCN2 has been shown to be have $97 \%$ homology (one amino acid different) to mouse UCN2 (Rademaker et al. 2005). UCN2 was injected i.v. as two incremental bolus doses of 25 and $100 \mu \mathrm{g}$ given $2 \mathrm{~h}$ apart.

Arterial pressure recordings, using an in-house online data acquisition system, commenced $30 \mathrm{~min}$ before the first injection and were continued for $120 \mathrm{~min}$ after the high dose. Heart rate and pressures were digitally integrated in 5-min recording periods, and data were recorded at preset intervals throughout the study. Cardiac output (thermodilution) was measured in triplicate (three values within 10\%) at preset intervals for the duration of infusions. Calculated total peripheral resistance (CTPR) was calculated as mean arterial pressure (MAP) divided by cardiac output. Stroke volume was calculated as cardiac output divided by heart rate.

Venous blood was drawn at preset intervals during the study protocol. Blood was taken into chilled EDTA tubes, centrifuged, and the plasma stored at $-80^{\circ} \mathrm{C}$ before assay for catecholamines (Goldstein et al. 1981).

\section{Ganglionic blockade}

Six additional sheep, surgically prepared as above but without usable nerve recording fields, were used to assess the effect of ganglionic blockade on UCN2-induced rises in heart rate. After 30-min baseline recording, the sheep received i.v. hexamethonium at a dose previously shown to inhibit CSNA (Jardine et al. 2002) namely $120 \mathrm{mg}$ hexamethonium infused over $60 \mathrm{~min}$. Thirty minutes after commencement of hexamethonium, the sheep received an i.v. bolus $(25 \mu \mathrm{g})$ of UCN2. Heart rate, MAP, and cardiac output were recorded at 15-min intervals from $30 \mathrm{~min}$ prior to ganglionic blockade to 60 min after UCN2.

\section{Statistical analysis}

Results are expressed as mean \pm s.E.M. Two-way ANOVA with time as a repeated measure was used to determine time and treatment differences between UCN2 and control arms of the study. Where significant differences were identified by ANOVA, a priori Fisher's protected least square difference (LSD) tests were used to identify individual time-points significantly different from time-matched control data. One-way ANOVA with time as a repeated measure was used to determine significant effects of UCN2 in the hexamethonium-treated sheep. Where significant differences were identified by ANOVA, a priori Fisher's protected LSD tests were used to identify individual time-points significantly 
different from baseline values. T-tests were used to determine differences in maximal hemodynamic response to UCN2 between normal sheep and sheep treated with hexamethonium. Statistical significance was assumed at $P<0 \cdot 05$.

\section{Results}

All animals successfully completed the experimental protocol, and full data collection was achieved with the exception of plasma epinephrine measurements. In samples from two animals, the epinephrine peaks from the HPLC profiles of the catecholamine assays were masked by unknown interference. Therefore, plasma epinephrine data are shown for $n=5$ sheep. CSNA burst area is an area under the curve measurement calculated in arbitrary units, and as such these data are expressed as percentage change from baseline. Significant baseline (time-points -30 to $0 \mathrm{~min}$ ) differences were observed for CSNA burst frequency $(51 \cdot 9 \pm 7 \cdot 6$ vs $63.9 \pm 12 \cdot 7$ bursts/min for control and active days respectively, $P<0 \cdot 05)$ and CSNA burst incidence $(62 \cdot 8 \pm 6 \cdot 3$ vs $72 \cdot 3 \pm 9 \cdot 2$ bursts $/ 100$ beats for control and active days respectively, $P<0 \cdot 05)$. Therefore, these data are also presented as percentage change from baseline.

Original arterial pressure, integrated CSNA, and ECG recordings from a representative sheep are shown in Fig. 1. Compared with baseline (top left panel), $30 \mathrm{~min}$ following $25 \mu \mathrm{g}$ bolus (top right panel), UCN2 induced a reduction in arterial pressure and increase in heart rate with modest increase in CSNA burst frequency (corresponding to the increased heart rate), but no increase in burst amplitude. In the lower panels, $90 \mathrm{~min}$ following $25 \mu \mathrm{g}$ bolus (lower left) or $100 \mu \mathrm{g}$ bolus (lower right), the effects of UCN2 on arterial pressure and heart rate were no longer evident, while dose-dependent and sustained reductions in both CSNA nerve frequency and amplitude were observed.

UCN2 induced dose-dependent prompt, but short-lived reductions in MAP $(P=0 \cdot 016$; Fig. 2$)$. In response to both doses, the nadir in MAP was $30 \mathrm{~min}$ after the bolus with pressures returning to time-matched control by $60-\mathrm{min}$ postbolus. Dose-dependent increases in heart rate $(P<0 \cdot 001)$ were longer lasting, and while the peak response still occurred at $30 \mathrm{~min}$ after each bolus, heart rate remained above timematched control at least $120 \mathrm{~min}$ after each dose (Fig. 2). UCN2 also increased cardiac output $(P<0 \cdot 001)$, to a similar degree with both doses with outputs only returning to timematched control values after $90 \mathrm{~min}$ following each bolus (Fig. 2). Increases in cardiac output were reciprocated by falls in CTPR $(P<0 \cdot 001)$, with changes following the same time-course as observed for cardiac output (Fig. 2). Stroke volume fell following both doses of UCN2 and remained lower than time-matched control for the duration of the study $(P<0 \cdot 001)$.

UCN2 induced dose-dependent changes in all indices of CSNA compared with time-matched control. CSNA burst frequency (burst/min) showed a biphasic response $(P<0 \cdot 001)$ with an acute rise above vehicle control confined to the first 15-30 min after $25 \mu \mathrm{g}$ bolus followed by a sustained reduction below vehicle control at 90-120 min following the UCN2 bolus (Fig. 3). Administration of the $100 \mu \mathrm{g}$ bolus also induced a brief (15 min) rise in burst frequency followed by a sustained reduction. CSNA burst incidence (bursts/ 100 beats) did not show this biphasic response but rather a significant $(P<0 \cdot 001)$ and progressive reduction to remain $\sim 40 \%$ lower than time-matched vehicle control for $120 \mathrm{~min}$ following the high dose bolus of UCN2 (Fig. 3). Similarly,
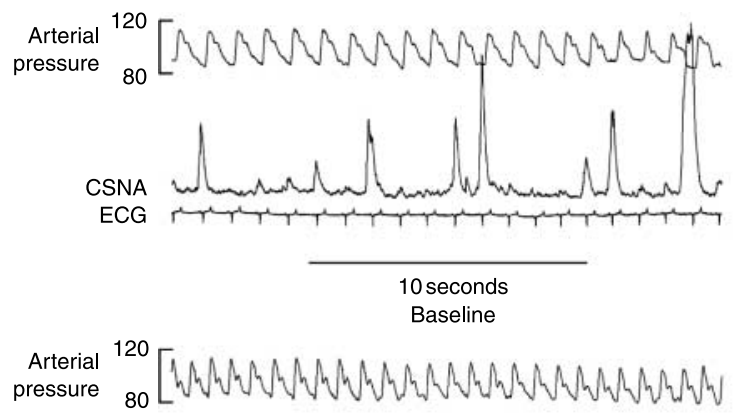
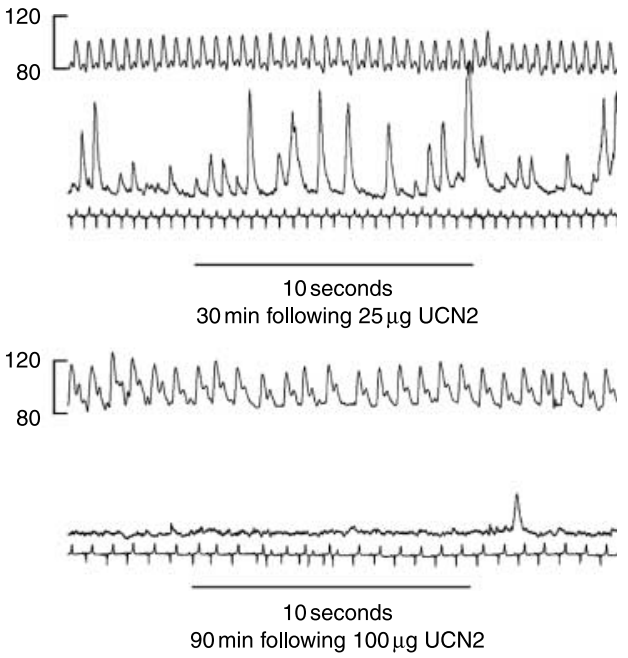

Figure 1 Sample tracings from a representative sheep of arterial pressure, cardiac sympathetic nerve activity (CSNA), and ECG at baseline, 30 and $90 \mathrm{~min}$ following $25 \mu \mathrm{g}$ bolus and $90 \mathrm{~min}$ following $100 \mu \mathrm{g}$ bolus administration of urocortin 2 (UCN2). 


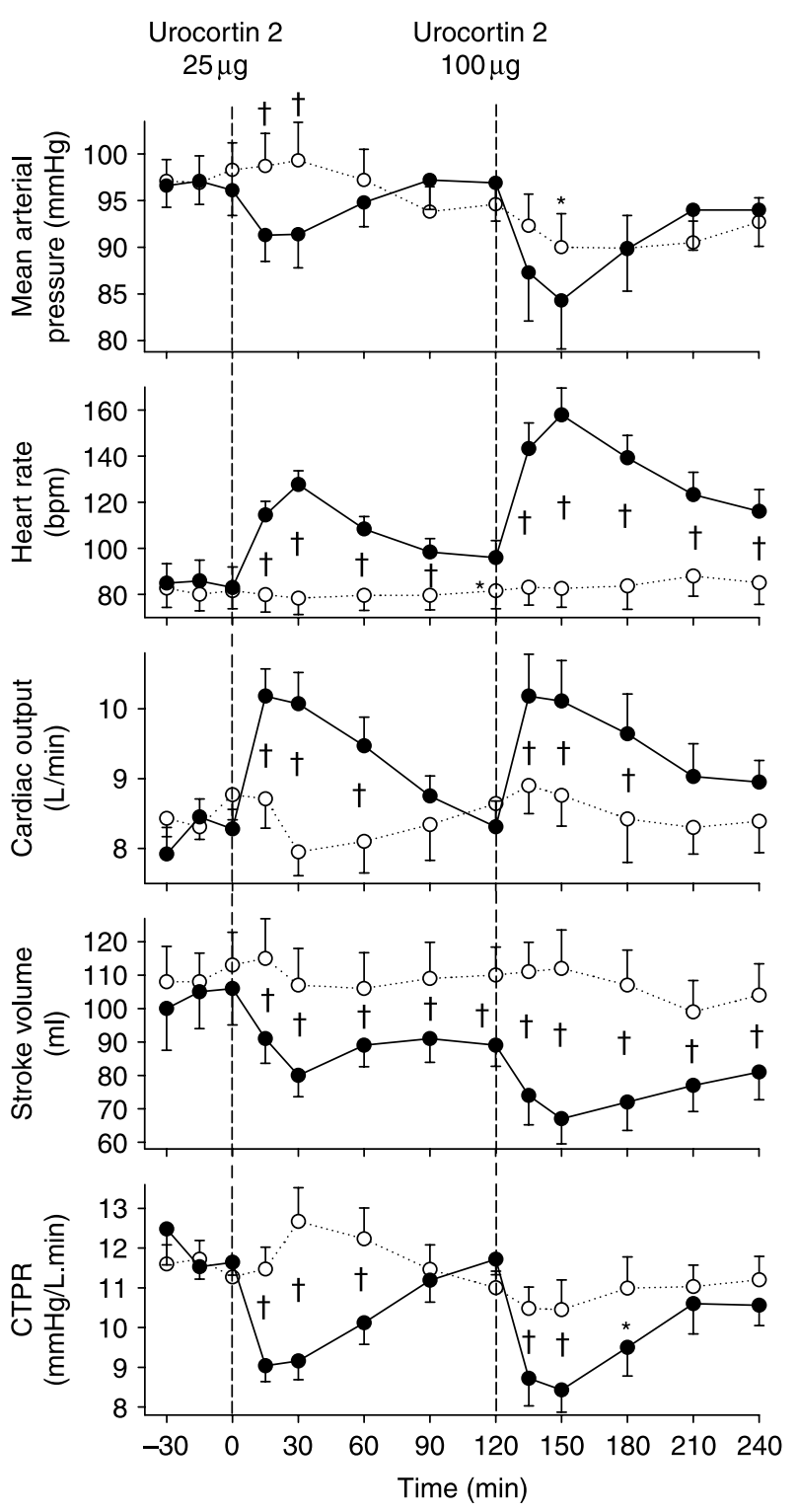

Figure 2 Mean arterial pressure, heart rate, cardiac output, stroke volume, and calculated total peripheral resistance (CTPR) response to i.v. bolus administration of urocortin $2(\mathbf{)})$ at doses of 25 and $100 \mu \mathrm{g}$ or vehicle control $(O)$ in seven sheep. Values shown are mean \pm S.E.M. Urocortin 2 induced significant falls in mean arterial pressure $(P=0 \cdot 016)$, stroke work $(P<0 \cdot 001)$, and in CTPR $(P<0 \cdot 001)$ and significant rises in heart rate $(P<0 \cdot 001)$ and cardiac output $(P=0 \cdot 03)$. Individual time-points significantly different from time-matched control (Fisher's protected LSD) are indicated by ${ }^{*} P<0 \cdot 05$ and ${ }^{\dagger} P<0 \cdot 01$.

both CSNA burst area/min $(P<0 \cdot 001)$ and burst area/ 100 beats $(P<0 \cdot 001)$ were dose-dependently reduced in response to bolus administration of UCN2 with levels being 50-80\% lower than time-matched vehicle control following the $100 \mu \mathrm{g}$ UCN2 bolus (Fig. 3).
Bolus administration of UCN2 at these doses induced significant, short-lived activation of plasma norepinephrine $(P=0 \cdot 006)$, with significant elevation of levels occurring 15-30 min following each UCN2 bolus administration but thereafter norepinephrine returned to time-matched control levels (Fig. 4). Plasma epinephrine levels $(n=5)$ tended to follow a similar pattern but these changes did not achieve statistical significance (Fig. 4).

\section{Ganglionic blockade}

Ganglionic blockade with i.v. hexamethonium induced minimal rises in heart rate and minimal reductions in MAP, cardiac output, and stroke volume with only the effect on stroke volume being statistically significant (Fig. 5). Subsequent bolus administration of UCN2 resulted in significant rises in heart rate within $15-30 \mathrm{~min}$ of UCN2 bolus $(P<0 \cdot 001)$. UCN2 induced significant falls in MAP ( $P$ - 0.018$)$, stroke volume $(P<0.001)$, and CTPR $(P<0 \cdot 001)$ but no consistent effect on cardiac output in hexamethonium-treated sheep (Fig. 5). Comparison of the maximal change in response to $25 \mu \mathrm{g}$ bolus of $\mathrm{UCN} 2$ for these hemodynamic indices in normal and hexamethonium-treated sheep is shown in Fig. 6. Maximum increase in heart rate was similar between the two groups of sheep being $42 \cdot 4 \pm 11 \cdot 5$ and $52 \cdot 2 \pm 6.9$ in normal and hexamethonium-treated sheep respectively. Cardiac output increments were significantly attenuated in the hexamethonium sheep $(P=0 \cdot 02)$. There was no significant difference in the response of MAP, CTPR, or stroke volume between the two groups (Fig. 6).

\section{Discussion}

The effect of UCN2 on SNA, including sympathetic traffic to the heart, has not previously been reported. Accordingly, we have examined the effects of UCN2 on CSNA, hemodynamics, and plasma catecholamines in normal conscious sheep. Bolus i.v. administration of UCN2 resulted in the expected hemodynamic actions of transient falls in arterial pressure with more sustained rises in heart rate and cardiac output and falls in CTPR. CSNA burst frequency showed a biphasic response with an acute rise followed by a more prolonged fall. All other indices of CSNA showed prolonged, dose-dependent falls in response to UCN2. UCN2 induced a short-lived activation of plasma catecholamine levels. Thus, UCN2 exhibits potent sustained inhibition of sympathetic traffic to the heart, despite an apparent shortlived generalized baroreceptor-induced activation of sympathetic activity as indicated by plasma catecholamines. The concurrent pronounced activation of heart rate suggests a direct chronotropic action for UCN2, which appears to be (at least in part) independent of actions on the autonomic nervous system.

While studies in man and sheep from our laboratory have reported plasma catecholamine levels following systemic 


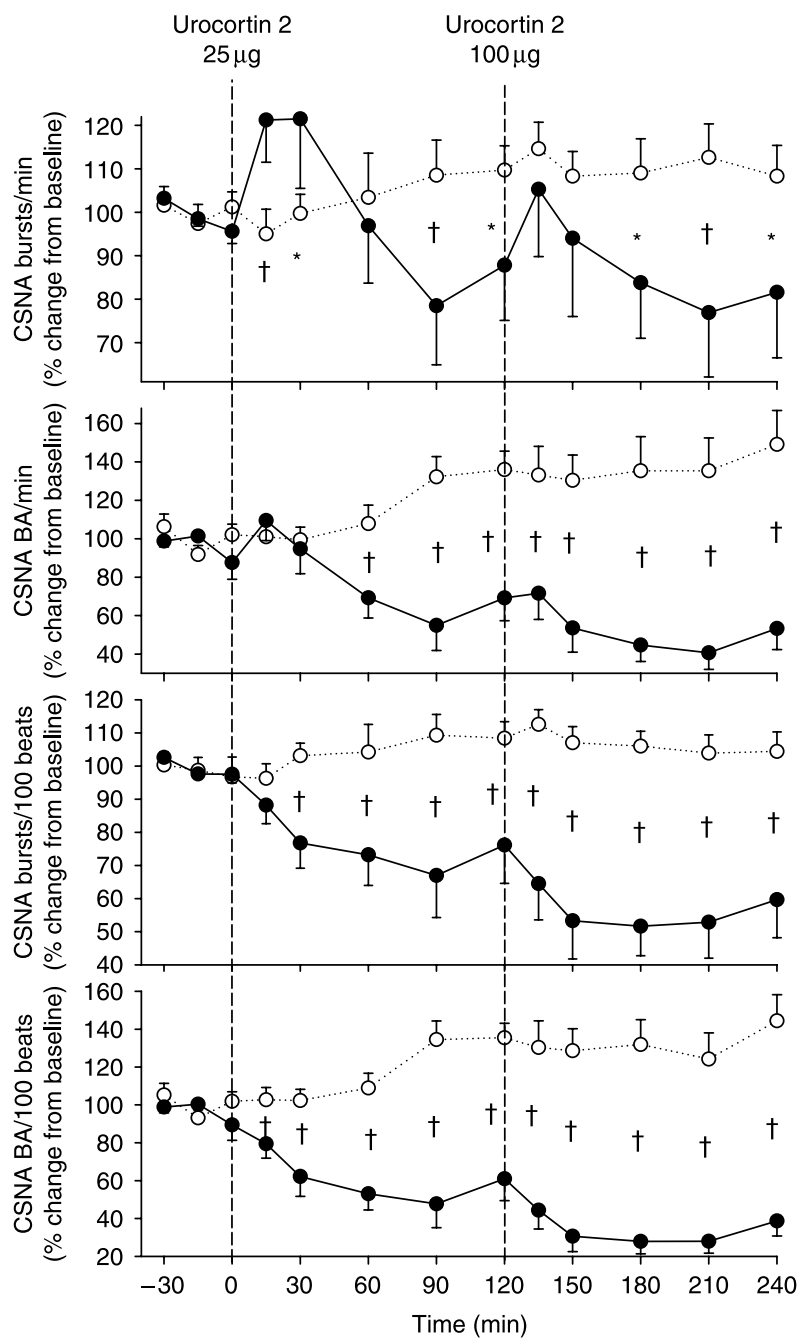

Figure 3 Cardiac sympathetic nerve activity (CSNA)

burst frequency (bursts/min), burst area (BA/min), burst incidence (bursts/100 beats), and burst area incidence (BA/100 beats) responses to i.v. bolus administration of urocortin $2(-)$ at doses of 25 and $100 \mu \mathrm{g}$ or vehicle control $(O)$ in seven sheep. Values shown are mean \pm S.E.M. Urocortin 2 induced a significant biphasic response in CSNA bursts/min $(P<0 \cdot 001)$ and consistent falls in $\mathrm{BA} / \mathrm{min}(P<0 \cdot 001)$, bursts/100 beats $(P<0 \cdot 001)$, and BA/100 beats $(P<0 \cdot 001)$. Individual time-points significantly different from time-matched control (Fisher's protected LSD) are indicated by ${ }^{*} P<0 \cdot 05$ and ${ }^{\dagger} P<0 \cdot 01$.

UCN2 administration, results have been variable. In normal and HF sheep, there were no significant changes observed in plasma norepinephrine levels, but plasma epinephrine levels were reduced following UCN2 administration in HF sheep (Rademaker et al. 2005). In normal humans, UCN2 induced a rise in circulating norepinephrine without change in epinephrine levels (Davis et al. 2007a), while identical doses of UCN2 infused in patients with stable HF resulted in no significant changes in either plasma epinephrine or norepinephrine (Davis et al. 2007b). Results from the present study clearly show that UCN2 raised plasma norepinephrine levels in a manner that reciprocated a significant fall in MAP. In fact, the time-course of changes in norepinephrine and in MAP were identical, with the peak or nadir respectively occurring 15-30 min after each dose. This pattern was identical for plasma epinephrine with a trend to rise, which did not achieve statistical significance, as results could only be obtained for $n=5$ sheep. In addition, the reciprocal changes in blood pressure and plasma norepinephrine suggest that the rise in plasma catecholamines reflect a baroreceptor-induced reflex response, probably directed at the peripheral vasculature. In vitro data suggest that UCN2 may regulate catecholamine synthesis and release in the adrenal medulla. In cultured PC12 cells, UCN2 induces norepinephrine release and phosphorylation of tyrosine hydroxylase through protein kinase $\mathrm{A}$ and protein kinase A-Erk1/2 pathways respectively (Nemoto et al. 2005). However, conversely, UCN2 activation of CRFR 2 actually suppresses the secretion of catecholamines in dispersed rat and human adrenal chromaffin cells, whereas UCN1/CRF activation of CRFR1 induces catecholamine secretion (Dermitzaki et al. 2007). On balance, it would appear that plasma catecholamine responses in the present study reflect a generalized baroreceptor-induced activation of the SNS.

Despite this apparent generalized activation of the SNS, the main finding of our study is that UCN2 induces prolonged and potent inhibition of sympathetic drive directed to the heart as measured by direct microneurography. In the case of

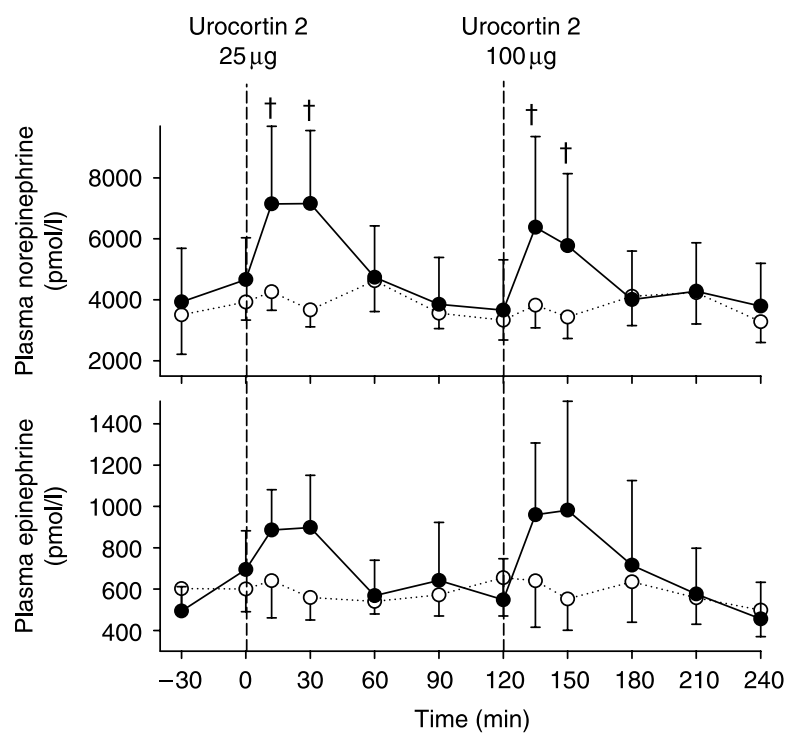

Figure 4 Plasma norepinephrine $(n=7)$ and epinephrine $(n=5)$ response to i.v. bolus administration of urocortin $2(\Theta)$ at doses of 25 and $100 \mu \mathrm{g}$ or vehicle control $(\bigcirc)$. Values shown are mean \pm S.E.M. Urocortin 2 induced significant rises in plasma norepinephrine $(P=0 \cdot 006)$. Individual time-points significantly different from time-matched control (Fisher's protected LSD) are indicated by ${ }^{+} P<0 \cdot 01$. 


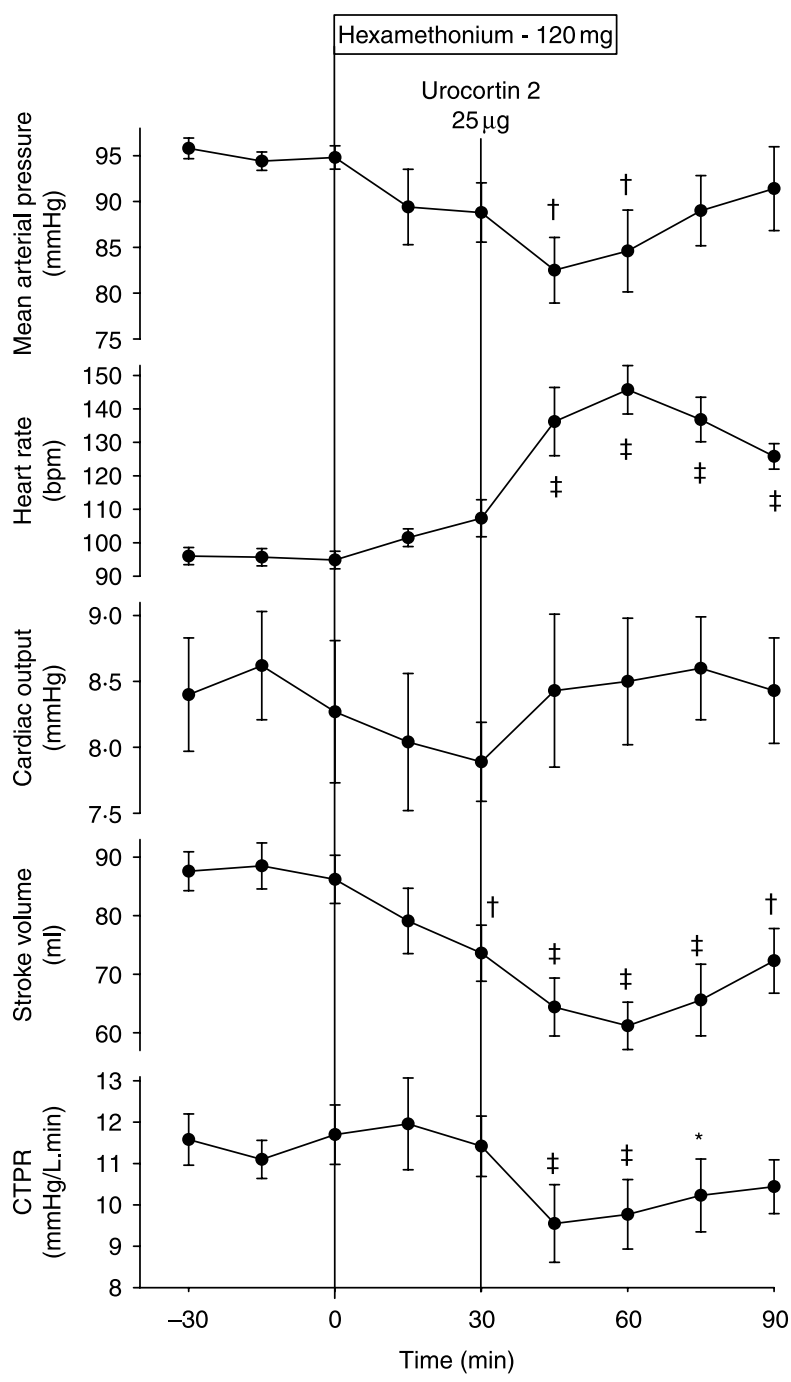

Figure 5 Hemodynamic response to i.v. bolus administration of urocortin $2(25 \mu \mathrm{g})$ in six sheep that received the ganglionic blocking agent hexamethonium (120 mg over $60 \mathrm{~min}$ ). Values shown are mean \pm S.E.M. Urocortin 2 induced significant falls in mean arterial pressure $(P=0 \cdot 18)$, stroke volume $(P<0 \cdot 001)$, and calculated total peripheral resistance (CTPR; $P<0 \cdot 001)$ and significant rises in heart rate $(P<0 \cdot 001)$. Individual time-points significantly different from baseline values (Fisher's protected LSD) are indicated by $* P<0 \cdot 05,{ }^{\dagger} P<0 \cdot 01$, and ${ }^{\ddagger} P<0 \cdot 001$.

CSNA burst frequency, the observed pattern of changes was biphasic, with an acute rise in burst frequency at 15-30 min following each dose of UCN2 followed by a sustained fall below time-matched control levels. All other indices of CSNA were reduced for the duration of measurements following UCN2 administration. Such falls in CSNA during concurrent activation of plasma catecholamines should not be interpreted as conflicting data, as it is important to note that there can be great selectivity of sympathetic reflex activity to different tissue beds (Ninomiya et al. 1971, Pagani et al. 1974).
This highlights the need to measure SNA efferent traffic to the organ of interest. We propose that the biphasic change in CSNA burst frequency may be explained by initial vasodilation and off-loading of baroreflex activity, contributing to the observed increase in HR and a short-lived increase in CSNA burst frequency. However, indices of CSNA burst size suggest simultaneous central inhibition of CSNA, which is of longer duration. Sympathetic nerve burst frequency reflects probability of firing of individual fibers, is gated to $\mathrm{HR}$, and is regulated primarily by baroreflex responses to rapid changes in blood pressure (Sundlof \& Wallin 1978, Malpas \& Ninomiya 1992a). However, burst size, reflecting recruitment of postganglionic fibers, is more affected by central pathways, for example, paraventricular nucleus secretion of vasopressin (Malpas \& Coote 1994) and chemoreceptor stimulation (Malpas \& Ninomiya 1992b). Therefore, the temporal changes we observed in different CSNA indices may be explained by the immediate indirect effects of baroreflex unloading coupled with more persistent direct sympathoinhibitory effects of UCN2 on the CNS. Nonetheless, the initial acute activation of CSNA burst frequency observed for UCN2 in the present study is in contrast to that previously observed for UCN1, where there was also no other evidence for other activation of the SNS as measured by plasma catecholamines (Charles et al. 2008).

The mechanism underlying UCN2-induced inhibition of CSNA cannot be defined by the present study but presumably occurs via central pathways. Of note, UCN2 has been shown to cross the blood-brain barrier in intact form by passive diffusion, entering brain parenchyma at a moderate rate (Kastin \& Akerstrom 2002). The neuroanatomy of sympathetic outflow is well known, in that the hypothalamus sends both direct and indirect projections, via the brainstem, to sympathetic preganglionic neurons in the spinal cord (Saper et al. 1976). Regional expression of the UCNs
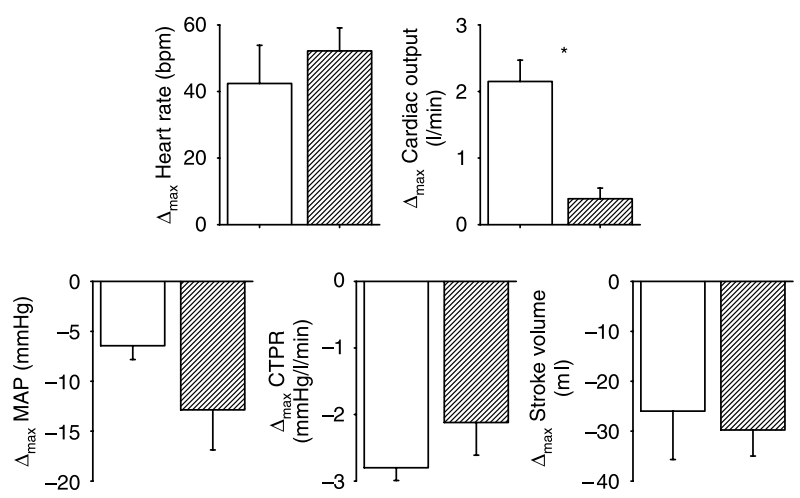

Figure 6 Comparison of maximum change in hemodynamic parameters in response to $25 \mu \mathrm{g}$ urocortin 2 in normal sheep (open bars, $n=7$ ) compared with ganglionic-blocked sheep (pretreatment with hexamethonium; crosshatched bars, $n=6$ ). Values shown are mean \pm S.E.M. There was a significant difference between the response of cardiac output between the normal and hexamethonium group $\left({ }^{*} P=0 \cdot 02\right)$. 
throughout the brain suggests a neurotropic role for these peptides in the CNS (Koob \& Heinrichs 1999). Furthermore, $\mathrm{CRF}_{2}$ receptors are present at high levels in the CNS, including autonomic regulatory centers in the hypothalamus and brain stem (De Fanti \& Martinez 2002). Although the above-mentioned studies have shown that pathways exist whereby both central and systemic administration of UCN peptides can activate neuronal projections throughout the hypothalamus, brainstem, and spinal cord and could thereby modulate sympathetic outflow, further studies are required to confirm such a mechanism.

In the present study, UCN2 clearly induced acute falls in MAP. This appears to be in contrast to our previously published data on UCN2 in normal sheep where we reported a subtle but overall significant rise in MAP in response to similar doses of UCN2 (Rademaker et al. 2005). However, careful examination of the time-course of the changes in MAP in our earlier study (Rademaker et al. 2005) shows that MAP was only raised above time-matched control data from 45 min following bolus administration and then only by 3-4 mmHg. In fact, the acute effect (at 15-30 min following bolus administration) was a trend (not significant) for MAP to fall, as seen in the present study. Significant falls in MAP (15-30 min following UCN2 bolus) were observed in the sheep with pacing induced HF (Rademaker et al. 2005). Thus, in our earlier publication (Rademaker et al. 2005), the overall small elevation of MAP reported for normal sheep masked an initial transient fall in MAP.

Falls in MAP in the present study were reciprocated by rises in heart rate. However, while falls in MAP were short lived (15-30 min postbolus), heart rate remained significantly above time-matched control for the duration of the study (at least 120 min postbolus). This may indicate that rises in heart rate in response to UCN2 are not merely baroreceptor mediated but that UCN2 may have direct chronotropic actions to increase heart rate independent of baroreceptor activation. Indeed, we have previously shown that UCN2 induces large dose-dependent increases in heart rate in normal sheep in the absence of any fall in arterial pressure (Rademaker et al. 2005). These results are in contrast to those of Gardiner et al. (2007) who demonstrated that UCN2 (stresscopin-related peptide) administered to conscious male Sprague-Dawley rats lowered blood pressure and increased heart rate, but that the tachycardic effects were abolished by pretreatment with atropine and propanolol. Thus, Gardiner et al. (2007) concluded that in conscious male rats, the tachycardic effects of UCN2 (and UCN1) are due to autonomic nervous activation mainly through baroreflex mechanisms. However, there appears to be species differences to the effects of the UCN peptides on heart rate and whether or not they are only mediated by baroreceptor activation following falls in arterial pressure. There may also be other factors contributing to the tachycardic effects of the UCN peptides (for example gender) but this requires further study. With regard to a role for the autonomic nervous system, in the case of UCN1, rises in cardiac output, heart rate, and cardiac contractility were similar in intact sheep and in sheep undergoing ganglionic blockade with hexamethonium (Parkes \& May 1999). Thus, it seems likely that, at least in conscious sheep, the UCN peptides may have direct chronotropic actions independent of baroreceptor-mediated sympathetic activation. Of note, in the six sheep that underwent ganglionic blockade (hexamethonium infusion), the rise in heart rate following a $25 \mu \mathrm{g}$ bolus of UCN2 was $\sim 50 \mathrm{bpm}$ (Fig. 6), similar in magnitude to that observed in intact sheep. Thus, both UCN1 (Parkes \& May) and UCN2 (present study) dramatically increase the heart rate similarly in both intact sheep and ganglion blocked (hexamethonium) sheep. This indicates that the actions of the UCN peptides to increase heart rate are not mediated by the autonomic nervous system, and it is likely that they are mediated by a direct action of UCN peptides on the heart. We cannot discount a contribution from an action of the UCN peptides to inhibit vagal activity, although to our knowledge no studies have addressed this question to date. However, it should be noted that vagal withdrawal is likely to induce only a modest rise in heart rate in sheep. Previous studies both from our laboratory (unpublished data) and from the literature (Hays \& Webster 1971, Lumbers \& Yu 1999) show that i.v. atropine or vagal section increases heart rate in sheep by $\sim 20-30 \mathrm{bpm}$ with the maximum heart rates observed following withdrawal of vagal tone being 110-120 bpm. This is much less than the maximal increments observed $(80 \mathrm{bpm})$ to increase heart rate to $\sim 130$ and $180 \mathrm{bpm}$ following 25 and $100 \mu \mathrm{g}$ UCN2 respectively (Fig. 2).

In the present study, stroke volume fell following UCN2 administration. The observed reduction in stroke is likely to be secondary to the marked cardiac sympathetic drive. However, despite such dramatic cardiac sympathoinhibition, stroke volume did not fall precipitously, which may reflect some degree of inotropic action of UCN2, as has been suggested previously (Bale et al. 2004, Rademaker et al. 2005). Despite this reduction in stroke volume, cardiac output was actually increased following UCN2. Thus, the contribution of the dramatically increased heart rate to increase cardiac output more than counterbalanced the modest fall that would have been expected with this degree of stroke volume reduction. Of note, similar reductions in stroke volume but increments in cardiac output have previously been reported for UCN1 (Parkes \& May 1999). In hexamethonium-infused sheep, there was no significant increase in cardiac output in response to UCN2 but, of note, trend for cardiac output to fall with hexamethonium was reversed with administration of UCN2. Nonetheless, there was a relative lack of any contribution from positive inotropic actions of UCN2 in the pharmacologically denervated sheep. Taken together, any peptide that concurrently inhibits CSNA yet has direct actions on the heart to maintain cardiac output (and coronary blood flow) is of potential therapeutic interest in cardiovascular disorders. UCN2 is reported to reduce damage in rat hearts subjected to regional ischemia/reperfusion, probably via MAPK-dependent pathway (Brar et al. 2004). In the intact 
animal, reduced sympathetic traffic to the heart could further enhance these cardioprotective actions of UCN2 (as reviewed by Fekete \& Zorilla (2007) and Boonprasert et al. (2008)), particularly in the setting of acute myocardial injury and may also protect against incidence and/or severity of arrhythmias.

In conclusion, this is the first report of UCN2 effects on SNA and indicates potent inhibition of sympathetic traffic to the heart, despite a generalized baroreceptor-induced activation of sympathetic activity. It also confirms a potent action of UCN2 to increase heart rate in both intact and ganglion-blocked sheep. These findings suggest an important role for UCN2 in cardiovascular homeostasis and warrant further investigation for potential therapeutic applications in acute myocardial injury and heart disease.

\section{Declaration of interest}

The authors declare that there is no conflict of interest that could be perceived as prejudicing the impartiality of the research reported.

\section{Funding}

This work was supported by the National Heart Foundation of New Zealand, Lotteries Health Research, and Health Research Council of New Zealand.

\section{Acknowledgements}

We are grateful to staff of the University of Otago, Christchurch Animal Research Area for assistance with animal studies and Christchurch Cardioendocrine Laboratory staff for hormone assays.

\section{References}

Bale TL, Hoshijima M, Gu Y, Dalton N, Anderson KR, Lee KF, Rivier J, Chien KR, Vale WW \& Peterson KL 2004 The cardiovascular physiologic actions of urocortin II: acute effects in murine heart failure. PNAS 101 3697-3702

Boonprasert P, Lailerd N \& Chattipakorn N 2008 Urocortins in heart failure and ischaemic heart disease. International Journal of Cardiology 127 307-312.

Brar BK, Jonassen AK, Egorina EM, Chen A, Negro A, Perrin MH, Mjos OD, Latchman DS, Lee KF \& Vale W 2004 Urocortin-II and urocortin-III are cardioprotective against ischemia reperfusion injury: an essential endogenous cardioprotective role for corticotrophin releasing factor receptor type 2 in the murine heart. Endocrinology 145 24-35.

Charles CJ, Rademaker MT \& Richards AM 2004 Urocortins: putative role in cardiovascular disease. Current Medicinal Chemistry - Cardiovascular and Hematological Agents 2 43-47.

Charles CJ, Jardine DL, Nicholls MG, Rademaker MT \& Richards AM 2008 Urocortin 1 exhibits potent inhibition of cardiac sympathetic nerve activity in conscious sheep. Journal of Hypertension 26 53-60.

Davis ME, Pemberton CJ, Yandle TG, Fisher SF, Lainchbury JG, Frampton CM, Rademaker MT \& Richards AM 2007a Urocortin 2 infusion in healthy humans. Journal of the American College of Cardiology 49 461-471.
Davis ME, Pemberton CJ, Yandle TG, Fisher SF, Lainchbury JG, Frampton CM, Rademaker MT \& Richards AM $2007 b$ Urocortin 2 infusion in human heart failure. European Heart Journal $\mathbf{2 8}$ 2589-2597.

De Fanti BA \& Martinez JA 2002 Central urocortin activation of sympathetic-regulated energy metabolism in Wistar rats. Brain Research $93037-41$.

Dermitzaki E, Tsatsanis C, Minas V, Chatzaki I, Charalampopoulos I, Venihaki M, Androulidaki A, Lambropoulou M, Spiess J, Michalodimitrakis E et al. 2007 Corticotropin-releasing factor (CRF) and the urocortins differentially regulate catecholamine secretion in human and rat adrenals, in a CRF receptor type-specific manner. Endocrinology 148 1524-1538.

Fekete EM \& Zorilla EP 2007 Physiology, pharmacology, and therapeutic relevance of urocortins in mammals: ancient CRF paralogs. Frontiers in Neuroendocrinology 28 1-27.

Gardiner SM, March JE, Kemp PA \& Bennett T 2007 A comparison between cardiovascular actions of urocortin 1 and urocortin 2 (stresscopin-related peptide) in conscious rats. Journal of Pharmacology and Experimental Therapentics 321 221-226.

Goldstein DS, Feuerstein G, Izzo JL, Kopin IJ \& Keiser HR 1981 Validity and reliability of liquid chromatography with electrochemical detection for measuring plasma levels of norepinephrine and epinephrine in man. Life Sciences 28 267-275.

Hays FL \& Webster AJF 1971 Effects of cold, eating, efferent nerve stimulation and angiotensin on heart rate in sheep before and after autonomic blockade. Journal of Physiology 216 21-38.

Hsu SY \& Hsueh AJ 2001 Human stresscopin and stresscopin-related peptide are selective ligands for the type 2 corticotropin-releasing hormone receptor. Nature Medicine 7 605-611.

Jardine DL, Charles CJ, Melton IC, May CN, Forrester MDE, Frampton CM, Bennett SI \& Ikram H 2002 Continual recording of cardiac sympathetic nerve activity in conscious sheep. American Journal of Physiology 282 H93-H99

Kastin AJ \& Akerstrom V 2002 Differential interactions of urocortin/ corticotrophin-releasing hormone peptides with the blood-brain barrier. Neuroendocrinology 75 367-374.

Koob GF \& Heinrichs SC 1999 A role for corticotrophin releasing factor and urocortin in behavioural responses to stressors. Brain Research $\mathbf{8 4 8}$ 141-152.

Lewis K, Li C, Perrin MH, Blount A, Kunitake KS, Donaldson C, Vaughan J, Reyes TM, Gulyas J, Fischer W et al. 2001 Identification of urocortin III, an additional member of the corticotrophin-releasing factor (CRF) family with high affinity for the CRFR2 receptor. PNAS $987570-7575$.

Lumbers ER \& Yu ZY 1999 A method for determining baroreflex-mediated sympathetic and parasympathetic control of the heart in pregnant and non-pregnant sheep. Journal of Physiology 515 555-566.

Malpas SC \& Coote JH 1994 Role of vasopressin in sympathetic response to paraventricular nucleus stimulation in anesthetized rats. American Journal of Physiology 266 R228-R236.

Malpas SC \& Ninomiya I 1992a The amplitude and periodicity of synchronized renal sympathetic nerve discharges in anesthetized cats: differential effect of baroreceptor activity. Journal of the Autonomic Nervous System 40 189-198.

Malpas SC \& Ninomiya I $1992 b$ Effect of chemoreceptor stimulation on the periodicity of renal sympathetic nerve activity in anesthetized cats. Journal of the Autonomic Nervous System 37 19-28.

Nemoto T, Mano-Otagiri A \& Shibasaki T 2005 Urocortin 2 induces tyrosine hydroxylase phosphorylation in PC12 cells. Biochemical and Biophysical Research Communications 330 821-831.

Ninomiya I, Nisimaru N \& Irisawa H 1971 Sympathetic nerve activity to spleen, kidney and heart in response to baroreceptor input. American Journal of Physiology 221 1346-1351.

Pagani M, Schwartz PJ, Banks R, Lombardi F \& Malliani A 1974 Reflex responses to sympathetic preganglionic neurones initiated by different cardiovascular receptors in spinal animals. Brain Research 68 215-225. 
Parkes D \& May C 1999 Cardiac and vascular actions of urocortin. In Hormones and the Heart in Health and Disease, pp 39-52. Ed. L Share. Humana Press: NJ, USA.

Rademaker MT, Charles CJ, Espiner EA, Fisher S, Frampton CM, Kirkpatrick CM, Lainchbury JG, Nicholls MG, Richards AM \& Vale WW 2002 Beneficial hemodynamic, endocrine and renal effects of urocortin in experimental heart failure: comparison with normal sheep. Journal of the American College of Cardiology 40 1495-1505.

Rademaker MT, Cameron VA, Charles CJ \& Richards AM 2005 Integrated hemodynamic, hormonal and renal actions of urocortin 2 in normal and paced sheep: beneficial effects in heart failure. Circulation 112 3624-3632.

Rademaker MT, Cameron VA, Charles CJ \& Richards AM 2006 Urocortin 3: hemodynamic, hormonal and renal effects in experimental heart failure. European Heart Journal 27 2088-2098.

Reyes TM, Lewis K, Perrin MH, Kunitake KS, Vaughan J, Arias CA, Hogenesch JB, Gulyas J, Rivier J, Vale WW et al. 2001 Urocortin II: a member of the corticotrophin-releasing factor (CRF) family that is selectively bound by type 2 CRF receptors. PNAS 98 2843-2848.
Saper CB, Loewy AD, Swanson LW \& Cowan WM 1976 Direct hypothalamo-autonomic connections. Brain Research 117 305-312.

Sundlof G \& Wallin BG 1978 Human muscle nerve sympathetic activity at rest: relationship to blood pressure and age. Journal of Physiology 274 621-637.

Wiley KE \& Davenport AP 2004 CRF2 receptors are highly expressed in the human cardiovascular system and their cognate ligands urocortins 2 and 3 are potent vasodilators. British Journal of Pharmacology $143508-514$

Received in final form 14 October 2009

Accepted 13 November 2009

Made available online as an Accepted Preprint 13 November 2009 